AVANT, Vol. XI, No. 2

ISSN: 2082-6710 avant.edu.pl/en

DOI: 10.26913/avant.2020.02.02

OPEN

Oaccess 2 îg

\title{
Neural-based vs. Enactive Approaches to Consciousness and Social Cognition
}

\author{
Zsuzsanna Kondor \\ Institute for Philosophical Research \\ Hungarian Academy of Sciences \\ kondorzs@gmail.com
}

Received 30 August 2018; accepted 16 July 2019; published 6 January 2020

\begin{abstract}
In the present paper, I will investigate how consciousness studies and theories of social cognition relate to each other, and suggest that despite the results of scientific research, both social cognition and consciousness can be better understood within a wider framework, i.e., not exclusively in terms of intra-cranial processes. I will attempt to illuminate the advantages of embracing embodied cognition in contrast with focusing exclusively on neural and/or representational mechanisms when consciousness and cognition are in question.

In my argumentation, I will first show the divergence of consciousness- and cognitive-theories on the one hand, and delineate attempts to reconcile these two branches of investigation, on the other hand. Then I will relate the model of cognitive phylogeny, as regards social cognition, and the models of the evolution of consciousness as it emerged in scientific literature. In conclusion, I will attempt to expose the advantages of widening the horizon of investigation towards embodied and enacted cognition.
\end{abstract}

Keywords: consciousness; social cognition; social brain; embodiment; enactivism

\section{Cognition and Consciousness}

Casting a glance at the fields of research of cognition and that of consciousness, we face a curious situation. In both cases, representational terminology is primarily applied, but in the case of cognition, the focus is on the mind's operations, according to the traditional setup, whilst the focus is on the brain when consciousness is in question. 


\subsection{The traditional scenery}

As it is referred to in a commonly-used textbook, "[c]ognition, or mental activity, describes the acquisition, storage, transformation, and use of knowledge. If cognition operates every time you acquire some information, place it in storage, transform that information, and use it ... then cognition definitely includes a wide range of mental processes", which covers perception, memory, imagery, language, problem solving, reasoning, and decision making (Matlin, 2013, p. 2).

According to the traditional view, we gain input from the outside thanks to perceptual capacities, and due to the mind's processes we produce output as a response to the external stimuli. It is widely held that in the 17 th century, the mind was understood either via the analogy of the camera obscura or that of the theatre. We can find both metaphors in the coetaneous literature, though it is far beyond the scope of this paper to examine the extent to which this is in line with the oft-mentioned Cartesian depiction of the mind. ${ }^{1}$ Nevertheless, the human mind is construed as being comparable with the camera obscura, i.e., our mind in some way reconfigures the information gained for further processing. According to Descartes, this mental act provides us with ideas. But Descartes himself explicitly differentiates two meanings of idea: (i) it can be the result of a mental act, and (ii) the representation of the mental act's object. $^{2}$ Thus, from early on, cognitive processes are related to representations providing room for ambiguity.

In the 1950s, when the interdisciplinary study of the mind known as cognitive science emerged, the computational approach to the mind was dominant in the field. Accordingly, cognition was considered as being "the internal transformation of structures of symbolic representations" (van Gelder, 1999, p. 251). Irrespective of the differences between cognitivism and connectionism, the mind was thought to be the faculty of basically computational processes. It is important to underscore that cognitive science preserved some characteristics of behaviorism, viz., the requirement of objectivity (the necessity of a third person perspective) and proposition-centeredness (Pléh, 2009). Cognition as a kind of computation can be either conscious or unconscious, and there was some question whether consciousness was worth

\footnotetext{
${ }^{1}$ Regarding camera obscura, consider Locke's An Essay Concerning Human Understanding (2.11.17) where he describes cognition with the analogy of a dark room. With Descartes, the situation is a bit ambiguous because he applied the analogy of camera obscura explicitly to the eye, though some aspects of camera obscura (viz., its selective and reconfigurational mechanisms) make the analogy applicable to human cognition as well. (Schmal, 2012, pp. 117-135)

${ }^{2}$ As he wrote in the Preface of his Meditations on First Philosophy: "there is an ambiguity here in the word 'idea.' Idea can be taken materially, as on operation of the intellect ... [and] can be taken objectively, as the thing is represented by that operation". (Descartes, 1995, p. 7) If we take into consideration his Treatise on Light (Descartes, 1985, p. 81), a rather confused picture emerges regarding what we should think an idea is. On the one hand it refers to a mental act, but it can represent the thing, the object of the mental act, and the cause of the act itself, on the other hand. As he wrote: "there may be a difference between the [i] sensation we have of light (i.e., the idea of light which is formed in our imagination by the mediation of our eyes) and [ii] what it is that produces this sensation within us (i.e., what it is in a flame or the sun that we call by the name "light)". (1995, p. 30)
} 
studying in relation to cognition, or whether it could provide something additional to the understanding of the computational machinery.

Consciousness, as compared with cognition, refers not directly to the process of how we acquire knowledge, but it rather emphasizes the process' reflective, self-related character at the beginning, and later function as a necessary background against which meaning is comprehensible. Historically, the English term consciousness emerged at the turn of the 17-18th centuries. Its meaning is rooted in Platonism and gained the sense of a self-referential knowledge thanks to Ralph Cudworth and a little later to John Maxwell.

Like the Greek 'syneidesis' and the Latin 'conscientia'..., 'consciousness' changed its meaning from 'knowing together with someone else' to 'knowing something for oneself': the person with whom I am privy to something is not someone else. Thus, 'conscientia' (and 'consciousness') came to be used as referring to knowledge... of one's own states and acts, that is, it became truly self-referential. (Thiel, 1991, p. 82)

Locke was familiar with Cudworth's work, and his theory of personality is based on the selfreferential nature of consciousness. ${ }^{3}$ Reflexive thinking, sensation, will, and personal identity are all possible because of consciousness. Of course, consciousness was not a topic of philosophical interest per se for a long time. In the early 20th century, James believed consciousness "is supposed necessary to explain the fact that things not only are, but get reported, are known" ([1904]1987, p. 1142). A few decades later, Merleau-Ponty defined consciousness in a similar sense as James: "what we call nature is already consciousness of nature, what we call life is already consciousness of life and what we call mental is still an object vis-a-vis consciousness" (1967, p. 184). ${ }^{4}$ Though James had already suggested a parallel between conscious states and brain processes by $1890,{ }^{5}$ conscious phenomena were not accessible for scientific inquiry for a long time. Thus, most scientists considered consciousness to be a "fuzzy, ill-defined domain whose subjectivity put it forever beyond the reach of objective experimentation" (Dehaene, 2014, pp. 16f.).

As an early exception, Sperry proposed a modified concept of consciousness in 1969. He did not share the dominant conviction of the time which considered consciousness as "an 'inner aspect' of the brain process, or as some kind of parallel passive 'epiphenomenon' or 'paraphenomenon' or other impotent by-product, or even to regard it as merely an artifact of semantics, a pseudoproblem" (Sperry, 1969, p. 533). Instead, according to his emergent interaction scheme, he suggested revising the traditional "stimulus-response concepts of central nervous control" and emphasized that "[f]or a full explanation of these gnostic functions

\footnotetext{
${ }^{3}$ As he put it: "For, since consciousness always accompanies thinking, and it is that which makes every one to be what he calls self, and thereby distinguishes himself from all other thinking things, in this alone consists personal identity" (Locke, 2.27.9).

${ }^{4}$ Later I will return to Merleau-Ponty's effort to relate consciousness and nature, i.e., the attempt to bridge the gulf between naturalist and intellectualist conceptions of the mind.

${ }^{5}$ As James put it: "ask ourselves whether, after all, the ascertainment of a blank unmediated correspondence, term for term, of the succession of states of consciousness with the succession of total brain-processes, be not the simplest psycho-physic formula, and the last word of a psychology which contents itself with verifiable laws, and seeks only to be clear, and to avoid unsafe hypotheses. Such a mere admission of the empirical parallelism will there appear the wisest course". (James, 1932, p. 182)
} 
(sensations, percepts, ideas, images, illusions, feelings, etc.), we are going to need, in addition, a further description and account of the higher order pattern activity in the cerebral process, the emergent properties of which are conceived to constitute the qualities of consciousness" (Sperry, 1969, p. 535). Despite the fact that there were some suggestions for how to reconcile the physical, biological and social as applied to consciousness, recent mainstream consciousness studies do not go beyond biology and certain evolutionary necessity.

\subsection{Reconciling cognition and consciousness}

Cognition as an umbrella term for different mental activities/capacities, and consciousness as a facilitator of human intelligence seem to be in close relation. However, the history of psychology, the conditions under which cognitive science was born, and representationalist heritage have all led to a peculiar situation within which cognition as symbol manipulation and consciousness as bound to the biological hardly fit a coherent theory of mind.

Hereinafter, in contrast to cognitive science and scientific approaches to consciousness, I will briefly outline how activity theory, phenomenology, and more recently, extended phenomenological-cognitive science relate cognition and consciousness.

\subsubsection{Activity and Being-in-the-world}

Bonie Nardi's activity theory ${ }^{6}$ (and criticism directed against Hutchins' theory of distributed cognition) nicely illuminates how cognition and consciousness are divided up amongst different research fields. Her theory reaches back to the Russian psychology and neuropsychology of the 1920s. (She relies heavily on Lev Vygotsky, Sergei L. Rubinsthein, Alexander R. Luria, and Alexei N. Leontev). Activity theory as a social theory of consciousness, unlike the Cartesian tradition and biology-centered neuroscience, considers consciousness "as [being] inextricably woven together with the social, the social being defined as interactions with other real people, as well as interactions with the tools other real people have designed and left for others as part of their culture" (Nardi, 1996, 65). It does not hold that the brain causes consciousness, but "rather it provides the physical matrix in which it can develop and change". It suggests you are what you do", i.e., our consciousness is shaped by the activities in which we are engaged in the broadest sense (p. 66). Nardi shares Vygotsky's view of consciousness.

Consciousness... is the active processes of the higher and lower psychological functions. Because these functions arise, develop and change within a social matrix ..., they cannot be seen as residing strictly 'under the skull' - consciousness is social, simultaneously beyond and within the individual. Because of externalization, there is not a one-way path from outer to inner activity; rather, inner activity gets concretized and made public through external activity (p. 69).

Activity theory considers actions as conscious goal-directed processes bearing a hierarchical structure (i). Goals or motives are hierarchically ordered, i.e., a certain goal can entail other

\footnotetext{
${ }^{6}$ Nardi's activity theory can remind us of interaction theory (Fiebich et al., 2016; De Jaeger et al., 2016).
} 
goals as they provide conditions which are necessary for its fulfilment. An activity's subject is a person engaged in acting in accordance with the activity's object/motive, and is made of actions and operations. Operations "provide an adjustment of actions to current situations". All components of an action can vary dynamically in accordance with the circumstances. That is, goals can change variably during an action, and operations and actions may fluctuate in both directions depending on habituations and situations.

Activities are object-oriented (ii), where an object can be broadly construed to be physical, biological, or even social. "Every motive is an object ... but there is another related sense of the word object, i.e., a prospective outcome toward which activity is directed, around which activity is coordinated, and which will be crystallized in a final form when the activity is complete" (p. 67).

All actions are accompanied by internalization and externalization (iii). This entails a constant transformation: internalization provides mental capacities by which external manipulation can be modelled without external change; while externalization makes internal capacities and activities manifest. The latter makes coordination amongst the subjects of the activity possible. Nardi emphasizes the importance of mediation (iv) by tools, be it physical or psychological. Tools shape the way we interact with the world, and "tools reflect the experience of other people" as well (p. 68). Finally, the principle of development (v) suggests viewing activity "temporally, over time", and not reduced to strictly local neuronal activation.

On the basis of these five principles, Nardi emphasizes that capturing consciousness in biological terms entails many traps and hinders an understanding of the importance of interaction. She calls attention to the difficulties of understanding the workings of language or any other socially grounded phenomena when consciousness is construed as strictly a biological phenomenon.

While it might be argued that a neuroscientist would of course think that language is important to consciousness, there is in fact an unambiguous divide between the two approaches: a theory that is profoundly biological does not find causality in the artificial. The emphasis on biology leads one away from the artificial. One never gets from brain architecture as cause to activity as cause. Activity never gets into the discussion at all. I argue that it is necessary to adopt the activity theory strategy of acknowledging activity as the primary shaper of consciousness, and then to get to the brain and biology (p. 69; emphasis added).

Nardi maintains that human nature is conceivable along the concepts of cognition and consciousness, and takes into consideration four "powerful" paradigms: neuroscience, cognitive science, activity theory, and distributed cognition. According to her analysis, consciousness as a biological phenomenon stands in the focus of neuroscience on the one hand, and as a social phenomenon it provides ground for activity theory on the other hand. Similarly, cognition as the manipulation of representations is the target of investigation for cognitive science, and cognition as a social phenomenon is the focus of distributed cognition as Hutchins construed it. However, in the latter case representationalism is as present as it is in cognitive science, unlike activity theory. Additionally, distributed cognition is expressed in representational terms as regards consciousness as well. That is, consciousness means having a representation 
of ourselves and our relations in our environment. ${ }^{7}$ In other words, Nardi seems to propose that despite its intentions, distributed cognition cannot fulfil its ends because it remains within the framework of representationalism. ${ }^{8}$

\subsubsection{The embodied mind}

There have been other attempts to reconcile cognition and consciousness. Next, I will briefly outline Merleau-Ponty's proposal on how the physical-biological and conscious-cultural are reconcilable, and then delineate radical embodied cognitive science's position as regards phenomenality/conscious experience and cognition based on, as Merleau-Ponty coined the term, incarnated/embodied experience.

Merleau-Ponty's three orders suggests a model of how the physical-biological and cultural can relate to each other on the basis of the embodied experience. He proposed "the notion of form would permit a truly new solution" (1967, p. 131) of how to relate nature to consciousness or "the synthesis of matter and idea" (p. 137). Forms (the term refers to Gestalt psychology) or structures describe the patterns according to which the three orders work. "The physical form is an equilibrium obtained with respect to certain given external conditions" (p. 145). In the case of vital structures, in contrast, "equilibrium is obtained, not with respect to real and present conditions, but with respect to conditions which are only virtual and which the system itself brings into existence" (p. 145). In the human order, there emerges a third dialectic. "For, between man and the physico-chemical stimuli, it [the human work] projects "useobjects" (Gebrauchobjekts)—clothing, tables, gardens — and "cultural objects"—books, musical instruments, language - which constitute the proper milieu of man and bring about the emergence of new cycles of behavior" (p. 162). This so-called dialectic in The Structure of Behavior is there to overcome the chasm between intellectualism and materialism, to describe how deeply intertwined the relation is between the organism and its milieu, and importantly, how the orders relate to each other. The organism is capable of forming its environment, and at the same time its environment affords and challenges the organism's capacities, and hence shapes it. In the case of orders, the dialectic describes the peculiar relation of not transcending the lower order but being internally related to it, and thus being capable of reshaping it. That is, the human order does not transcend the lower ones, but is internally related to them, and this internal relation makes it possible to reshape the environment, and thus generate new behavioral structures.

Consciousness plays a crucial role in this process because it is "the theater of all being" (Merleau-Ponty, 1964, p. 55) and is "the subject for every possible object" (p. 57). Value entered the scene already in the vital order-since the organism "constructs a stable milieu for itself"

\footnotetext{
${ }^{7}$ That is, consciousness is understood in a 17-18th century manner.

${ }^{8}$ Nardi highlights additional differences between activity theory and distributed cognition: the latter regards cognition as computation, and does not take into account the important difference between applying knowledge as a routine and producing knowledge; it overlooks the forming potential of tools regarding cognitive capacities; "scale[s] back person", i.e., does not count with her inventive capacity; commits category mistakes when it does not differentiate between cognition and coordination regarding personal and group properties; and does not take into account the role of personal motives.
} 
in accordance with its needs (Merleau-Ponty, 1967, p. 162) — but "[a]t this level there is no question yet of a real self-awareness or of intentional activity. Moreover, the organism's prospective capability is exercised only within defined limits and depends on precise, local conditions" (Merleau-Ponty, 1964, p. 4). The world as arranged and formed beyond the limits of reciprocal adjustments is only possible when consciousness enters the scene. Merleau-Ponty distinguished between perceptual and intellectual consciousness, ${ }^{9}$ and suggests that perceptual is the primordial. He proposed reconsidering what perception is when he suggested "[p]erception is not a science of the world, it is not even an act, a deliberate taking up of a position; it is the background from which all acts stand out, and is presupposed by them. The world is not an object such that I have in my possession the law of its making; it is the natural setting of, and field for, all my thoughts and all my explicit perceptions" (Merleau-Ponty, 2005, p. x). That is, perception does not provide us truths, (Merleau-Ponty, 1964, p. 14), but provides an immediate and direct relation between the perceived and its meaning for us (p. 15). Perception is the establishment upon which any kind of relation (be it linguistic, cultural, moral or esthetical) with the world can be built. Perception is "a kind of practical synthesis: I can touch the lamp, and not only the side turned toward me but also the other side" (p. 14).

The body plays a crucial role in perception: it provides perspective, ties the perceiving self to "a system of things" (p. 21), accommodates multimodal synthesis, yields perceptual fields and field of practice, and "as an active body capable of gestures, of expression, and finally of language, it turns back on the world to signify it" (p. 7). Because "the perceived object is by definition present and living", perception is "a privileged realm of experience". Accordingly, "the experience of perception is our presence at the moment when things, truths, values are constituted for us" (p. 25). ${ }^{10}$

Accordingly, thanks to our body via perception, we are in direct and active interplay with the world. Our experience is embodied because "far from my body's being for me no more than a fragment of space, there would be no space at all for me if I had no body" (Merleau-Ponty, 2005, p. 89); and even the meanings of words derive "from our experience as embodied subjects. In space itself independently of the presence of a psychophysical subject, there is no direction, no inside and no outside" (p. 182).

\subsubsection{Cognition and consciousness: inseparable}

Radical embodied cognitive science [RECS] (Chemero, 2009) or extended phenomenologicalcognitive science (Silberstein\&Chemero, 2012) are in line with Merleau-Ponty's suggestions, since both consider human beings as being in immediate and perpetual commerce with their environment. Under this framework, cognition is in no way a computational process which operates on representations. Rather, it suggests that "we can understand phenomenology and

\footnotetext{
${ }^{9}$ We should notice here that Merleau-Ponty suggested there were many different kinds of consciousness when he wrote in the Phenomenology of Perception, "there are several ways for the body to be a body, several ways for consciousness to be consciousness" (Merleau-Ponty, 2005, p. 109).

${ }^{10} \mathrm{Cf}$. recent efforts and suggested solutions regarding the nature of phenomenal qualities. In the 2nd part I will return to this question in my discussion of sensorimotor theory and Humphrey's proposal.
} 
cognition as inseparable and complementary aspects of coupled brain-body-environment systems" (Silberstein \&Chemero, 2012, p. 40). This anti-representationalist setting relies on embodied cognition, the ecological approach, and dynamic system theory.

This view eliminates the mind-body divide because "mind-body-behavior is simply one process"; as consciousness is not neural-based, the neural correlate of consciousness does not entail the hard problem; there is no need for reduction when quale is in question because phenomenological experience is conceived as being relational; and this conceptual setting does not contradict and is even frequently supported by scientific results ${ }^{11}$ (p. 41).

Because cognition "is the ongoing activity of a nervous system, body, and niche nonlinearly coupled to one another" 12 and thus "phenomenology and cognition are inseparable" (pp. 43 and 46), conscious experience is not separated from cognition.

Within the framework of RECS, Kiverstein (2016) provides a detailed account of how neural activity shows an inseparable relation between cognition and consciousness. He suggests, in accordance with RECS, that cognition, perception, and action are continuous, and that cognitive processes are "constituted by temporally extended, skilled and practical engagements of embodied agents with the environments they inhabit." (Kiverstein, 2016)

Kivertsein believes cognitivism's computational model of cognition encourages some philosophers to argue for an unbridgeable gulf between subconscious cognition and phenomenal consciousness. RECS, on the contrary, suggests that both conscious and non-conscious cognitive processes are self-organizing and "mutually constrain each other as dynamical processes evolving over different time scales".

Self-organization is a process within which order emerges from disorder. In this process, local and global properties are in a circular causal relation, i.e., the interactions of the elements form the system's global behavior and at the same time the result of this bottom-up pattern

\footnotetext{
${ }^{11}$ For a selected list of scientific findings in line with extended phenomenological-cognitive science see Silberstein \& Chemero 2012, pp. 43-46.

${ }^{12}$ In the philosophical literature we can find an explicit challenge of embodied cognition. Aizawa (2015) calls our attention to the need for clarification of what cognition and embodiment mean. He thinks embodied and extended cognitions are very often defined implicitly as behavior. However, psychologists consider cognition as being the cause of behavior. He underscores that if we accept that cognition is nothing other than behavior, as many theorists seem to suggest, then embodiment does not come into question, since traditional literature regards behavior as embodied (and behavior is hardly comprehensible without an executing body). He formulates an additional criticism when he proposes at least two kinds of embodiment conception. Embodiment (i) "mean[s] something like the view that cognition is realized by, constituted by, or supervenes upon parts of the body other than just the brain"; embodiment (ii) proposes that the same mechanisms are in play regarding perception and action as in the case of concept manipulation and reasoning, i.e., reason is shaped by brain, body and experience. (Aizawa 2015)
}

As regards the second, Merleau-Ponty's considerations provided the ground for embodiment (ii) and I use the term in this sense. This is not in contradiction with the key idea of RECS. The first objection touches upon the question of whether we can have access to mental phenomena without bodily or linguistic expression. (See Wittgenstein, 1958, pp. 41f., James, 1884, pp. 189f.) And, importantly, it seems to suggest the separation of cognitive and bodily processes, which we need to avoid according to the RECS. 
constrains the elements' degrees of freedom. This circular causality applies to the brain and the body in its engagement with affordances.

To show how circular causality can be applied to the relation between brain and body, we need to re-define what phenomenal experience is. Kiverstein suggests describing it in terms of selforganization and active engagement. Like the Heideggerian term Being-in-the-world, which expresses how the surrounding world emerges as a totality of references to help the orientation of acting humans, as opposed to the intellectualist account, thus suggesting we are primordially engaged in the world, we cope with things as ready-to-hand tools (Heidegger, 1962, p. 69f.). ${ }^{13}$ In RECS' terms we cope with affordances.

In accordance with self-organization and because of the inherent instability of self-organizing systems, this active engagement is driven by affective tension - which we need to reduceand is based on evolving skills (in a broad sense). There is a complex interplay between the agents' concerns and their ambient world (to use Heidegger's terms), or between interests and landscape of affordances, in RECS terms. ${ }^{14}$ And there is a continuous change in the state of both the agent and its environment. Engagements with affordances are inherently unstable. However, the whole system is characterized by metastability, i.e., an organism can maintain focus while at the same time there is room for shifting focus when necessary. Phenomenal experience is "emergent from this dynamical, self-organising process" (Kiverstein, 2016).

Similarly, neural synchronization is on a par with the changes in the brain-body-niche system. When disequilibrium increases, large scale synchronous activity shows up in the brain. This large scale synchronous neural activity is related to phenomenal consciousness and it is in line with action readiness, i.e., readiness to answer to relevant affordances. ${ }^{15}$ That is, Kivetrstein suggests, the changes in the states of the brain-body-niche system — which are continuous because of the inherent instability of self-regulating systems - in accordance with the system's metastability, are orchestrated according to robustness and flexibility which provide ground for fine tuning at each level. In this nesting structure, subconscious cognition and conscious perception are intertwined. As Kiverstein writes: "The integrated activity that unites systems from the highest to the lowest levels of organisation in the brain holds together because of the circular causal interactions that entrain the activity in the individual systems to a global pattern." (2016)

All the above-described approaches to consciousness suggested a certain continuum between consciousness and cognition, which is considered necessary in everyday activity; significantly, active commerce with both the physical and social environment is seen as indispensable. Similarly, all three above-mentioned approaches eschew mental representation as far as possible.

\footnotetext{
${ }^{13}$ Unlike entities around us emerging as present-at-hand things in the case of theoretical investigations or in case of "unusability". For details, see Heidegger, 1962, pp. 102 f.

${ }^{14}$ Accordingly, phenomenal experience is active. As Kiverstein highlighted, here we can find convergences with the sensorimotor approach. I will return to his question in the third part of the paper.

${ }^{15}$ For details regarding how large-scale synchrony is related to consciousness, see Dehaene (2014).
} 


\section{The Social Brain and the Evolution of Consciousness}

The following approaches focus on either cognitive evolution and its biological establishment, or the evolution of consciousness while focusing one-sidedly on intra-cranial processes and sticking to representationalism. First, I will quickly outline Robin Dunbar's social brain hypothesis and adumbrate a theory of cognitive evolution in a representationalist framework, where the latter suggests something very close to the extended mind hypothesis. Then, I will recapitulate Graziano's social attention schema and Humphrey's functionalist account of consciousness, as both consider consciousness in an evolutionary setting but focus on intracranial process and touch upon the question of qualia.

\subsection{The social brain hypothesis and cognitive evolution}

Dunbar's social brain hypothesis is based on a correlation between group size and the relative volume of the neocortex, i.e., it suggests that ever changing social relations entail an increasing computational burden proportional to the group size. The neocortical increase is supposed to have an external, environmental cause. As he wrote: "The social brain hypothesis implies that constraints on group size arise from the information-processing capacity of the primate brain, and that the neocortex plays a major role in this." (Dunbar, 1998, p. 184) Taking part in a group's life requires more than just the capability of remembering, it needs additional cognitive capacities - or in Dunbar's terms, various kinds of information processing. Additionally, recognizing and interpreting visual signals, identifying group-mates and behavior, remembering faces, relations within the group, being able to manipulate information about relations in the group, etc. are all a considerable load on memory.

Merlin Donald builds his cognitive evolutionary theory on the basis of Dunbar's social brain hypothesis, and does not suggest abandoning the representational account, though he calls attention to the importance of external scaffolding when cognitive evolution is in question. As he suggests: "Cultures restructure the mind, not only in terms of its specific contents, which are obviously culture-bound, but also in terms of its fundamental neurological organization". (Donald, 1993, p. 14) He differentiates four consecutive cultures. The first, episodic culture, is characterized by the episodic mind inherent in mammals and apes. Necessary procedural skills, perception, and episodic memory are adept, but "the limitations of episodic culture are in the realm of representation" (p. 160). In mimetic culture, representational skills such as mimicry and gestures emerge,${ }^{16}$ facilitating communication and group organization, paving the way for the more refined and effective representational skill of language. In the third phase of cognitive evolution, mythic culture, language emerges as "a wholly new system for representing reality" (p. 259). Linguistic capacity made creating mythical constructions possible, which served the community by providing orientation and structure. At the same time, language as a new representational form inserted a huge load on memory. The fourth distinguished period according to Donald, belongs to theoretical culture. The most significant

\footnotetext{
16 " [T] he mimetic mind models, in action, the outputs of the episodic mind. The mimetic system is thus a seminal hominid cognitive innovation, a mode of cognition that remains dissociable from language..." (Donald, 1993, p. 193)
} 
invention at this time is the external symbolic storage system. This seemingly technical invention entailed long-lasting modifications in cognitive organization beyond decreasing the memory load. ${ }^{17}$

Donald believes cave paintings emerged in oral-mythical culture, and that via the first pictorial images "a technological bridge was under construction that would eventually connect the biological individual with an external memory architecture." (p. 284) As we can see, Donald thinks of cognitive evolution in terms of evolving representational skills in an encapsulating manner. Though he takes external support into consideration as being capable of modifying cognitive operations, the emphasis is on the evolution of the representational repertoire of cognitive capacity.

\subsection{Evolutionary models of consciousness}

With his theory of attention schema, Michael S. A. Graziano hopes to explain how phenomenality emerges out of conscious experience. He thinks in terms of computation by the brain: "consciousness is not an emergent property, or a metaphysical emanation, but is itself information computed by an expert system". (Graziano \& Kastner, 2011, pp. 98f.)

Attention schema theory defines consciousness on the basis of social perception. The capability of modelling one's own attention can evolve on the basis of selective signal enhancement and the control of attention: "Awareness is a perceptual model of attention" (p. 100). The close relation between attention and awareness is in line with the fact that although they are not identical, most of the time they covary. This schema suggests that while attention selects among signals (as the brain is applying its "data-handling method"), the brain decides whether the selected signal will or will not entail awareness. This modelling capability makes it possible to predict behavior, not just one's own but also that of our companions. Monitoring one's own attention and social perception both provide ground for monitoring others' attention, hence, when awareness is attached to them, the schematic model of their attention enables the prediction of their behavior. Being aware is a feeling: "My awareness feels like it is located inside me. In a sense it is me" (p. 101).

In certain respects, Graziano's theory evokes higher-order monitoring and self-representational theories, and is not in contradiction with such influential theories as global workspace theory. And, importantly, it presumes social intelligence that "is the ability to compute information of the type, "Bill is aware of X". This attribution is based on our reconstruction of Bill's attention (p. 99). Graziano focuses on the brain's computation and despite the necessary condition of attention schema theory, viz., social interaction, he does not take into consideration extra-cranial conditions, neither body, nor environment, nor interaction with group mates

17 " [T] heoretic culture broke with the metaphoric style of meaning in oral-mythic culture. Where narrative and myth attribute significances, theory is not concerned with significance in the same sense at all. Rather than modeling events by infusing them with meaning and linking them by analogy, theory dissects, analyzes, states laws and formulas, establishes principles and taxonomies, and determines procedures for the verification and analysis of information. It depends for its advanced development on specialized memory devices, languages, and grammars" (Donald, 1993, pp. 274f.). For more detail regarding the difference between oral and literate expression of thought, see Kondor (2003). 
which can provide a kind of scaffolding for the further development of this modelling cognitive skills.

Like Graziano (and many others who are engaged in consciousness studies), Nicolas Humphrey attempted to reconcile qualia and brain-bound activities, i.e., to remove the obstacles to being able to consider mind and brain as "aspects of a simple state - a single state, in fact, of the material world" (2000, p. 5). According to the analogy of equations in physics, in order to be able to equate mental and brain states, we need to line up the two sides. In doing this, he applied Thomas Reid's brilliant but sadly forgotten distinction between sensation and perception. According to Reid, sensation's “very essence consists in being felt; and when it is not felt it is not ... Perception [by contrast] has always an external object; and the object of my perception, in this case, is the quality in the rose that I discern by the sense of smell" (Humphrey, 2008, p. 40).

In terms of functionality, Humphrey gives an evolutionary account of how simple cells could evolve into being capable of accommodating consciousness and describes how sensation evolved towards perception. Accordingly, the distinction between sensation and perception is based on their scopes and limits: sensation is "providing an affect-laden modality-specific body-centred representation of what the stimulation is doing to me and how I feel about it", while perception is "providing a more neutral, abstract, body-independent representation of the outside world" (Humphrey, 2000, p. 17). Though perception is built upon sensation, after their diremption, their evolution went on their own way.

Returning to the mind-brain equation, sensation is considered as being an activity. According to Humphrey "sensing is not a passive state at all, but rather a form of active engagement with the stimulus occurring at the body surface... I am reflexly reaching out to the body surface with an evaluative response ... it is this efferent activity that I am aware of. So that what I actually experience as the feeling ... is my reading of my own response to it" (Humphrey, 2000 , p. 13). If we consider sensory awareness as an activity, then we can equate it with neural activity in the brain.

For the mind side of the equation, our earlier analysis suggested that the experience of creating a sensation has many of the characteristics of creating a bodily expression. Now, for the brain side, we have constructed a history which suggests that sensations are the descendants of a kind of activity which once upon a time actually was a kind of bodily expression. ${ }^{18}$ True, the activity on the brain side nowadays is virtual, privatized, addressed to an as-if body; but there is every reason to suppose that its characteristics - its dimensions - have remained in line with what they were (2008, pp. 98f.).

\footnotetext{
${ }^{18}$ Cf. Adolphs' suggestion according to which our body is a "somatic scratchpad" enhancing brain processes. As we will see, Adolphs uses this metaphor to suggest including the body when cognition is in question, while Humphrey considers this function of the body evolving towards being modelled in the brain itself. I think that if we leave representationalism and accept that our body plays an important role in the formation of our brain capacity, this does not disqualify views suggesting the importance of bodily experiences even if Humphrey is right.
} 
Regarding qualia, sensation's action-like character provides phenomenality. Because sensation has a subject; refers to a bodily location; is always an ongoing present-tense feeling; because different feels can be different regarding their modality (visual, auditory, tactile, olfactory, and taste), and these four properties are always immediate (Humphrey 2000, 2008), it is capable of providing decisive properties of first person experience. Thus, sensations follow up our interaction with the world, create a sense of presence, of being engaged here and now, lending a kind of "me-ness" (2008, p. 70). ${ }^{19}$

In this second part of this paper, I have delineated three different theories of how cognition and consciousness could have evolved. These briefly outlined concepts follow the route of cognitive science, i.e., they frame cognition in representational terms and focus on the brain despite the evolutionary and social setting.

The social brain hypothesis, attention schema theory, and Humphrey's evolutionary approach to consciousness rely on representational skills and abandon the consideration of bodily engagement. Although Donald emphasizes the importance of external symbolic storage, he does not reach beyond some aspects of brain capacity (such as memory load) and continues focusing on representational skills. And while Humphrey's conciliation of brain activity and mental events suggests the activity-like character of sensation, it moves on the level of description, i.e., it describes the process in terms of action, but continues emphasizing the brain's simulating capacity as being a substitute for the evolutionally preceding bodily activity, and thus eliminates the body's role in the present.

\section{Stretching Beyond the Skull}

As we can see, the above-mentioned approaches to consciousness focus on skills that reside in the skull. Considering the brain as the source of social skills is apparently supported by some scientific evidence. For example, recent research in social cognition has detected that specific brain areas are responsible for skills needed in social interactions. For example, social cognitive neuroscience successfully relates neuronal activation patterns to social capabilities. In cases of brain lesions, certain areas (such as the prefrontal cortex, superior temporal sulcus, temporo-parietal junction, and amygdala) show the lack of conscious perception (as in case of neglect) and hinder skills which are morally and socially related (Graziano \& Kastner, 2011). A huge amount of scientific evidence suggests that "the brain is exquisitely tuned to respond to socially relevant signals with which it can interact" (Adolphs, 2007, p. 872). But these results provide evidence for the correlation between certain social behaviors and brain activity,

\footnotetext{
${ }^{19}$ Cases of blindsight demonstrate what happens when sensation is missing. Humphrey's experiences with a rhesus monkey whose visual striate cortex was removed and some human blindsighted patients proved that without conscious access to percepts, i.e., without sensation, visual experience cannot provide the same access to visual information as conscious experience does. Beyond the "tiresome duty" (2008, p. 69), the gained visual information is not worthy of interest because it is not related to the perceiving subject, it is hardly imaginable for the patients that they can see, if it turns out that they can see to some extent, they have no idea how it is possible, and furthermore, attributing seeing to someone else on the basis of their own visual experience is also not manageable. "Blindsight ... is affectless vison" — as Humphrey summarized (2008, p. 67).
} 
not for the claim that mere processes in the brain are sufficient to create consciousness and accommodate cognitive skills.

I will conclude by suggesting that we need to extend the scope of investigation beyond the skull and embrace embodied cognition. I propose the extension of the scope of investigation because this extension (i) makes obvious the continuity of consciousness, cognition, and behavior, (ii) paves the way to bridging the gap between the mental and physical, and (iii) facilitates the integration of interaction and context, which per se are implicated by social cognition and evolutionary approaches.

\subsection{Thought experiments and the burden of cognitivism}

Disembodied cognition was considered as a representation manipulating machinery. Computational processes do not entail consciousness; hence, cognition and consciousness belong to different conceptual realms. This separation is so deeply rooted that famous thought experiments are based on similar diremptions. For example, if we take into consideration the Chinese brain and Chalmers' zombie thought experiments, it is clearly evident that in both cases physical and functional identity does not warrant the same result. They suggest the neuron-like connectedness or a specific neural setting of a zombie, even the same neural setup as in a human being, does not create consciousness. In other words, though the same cognitive tasks can be executed, conscious experience is assigned intuitively only to humans. That is, as Dennett clearly summed up his criticisms, the zombie thought experiment suggests that we "can remove consciousness while leaving all cognitive systems intact" exactly in the same way as we could suppose "that by an act of stipulative imagination, you can remove health while leaving all bodily functions and powers intact" (Dennett, 1995, 325). ${ }^{20}$

As we can see, if we abandon the computational model of cognition, embrace RECS, and focus on interaction with the environment, the gulf between cognition and consciousness disappears. Casting a glance at consciousness studies, we can recognize that the focus on neural processes leads researchers to believe the key to consciousness is the brain and thus neural activity's relational character is limited to neural networks. The neural correlate of consciousness covers such key factors which are both phylo- and ontogenetically important. Consider what MerleauPonty and Nardi emphasized, i.e., a background against which humans are capable of forming their environment to the extent of bringing new behavioral patterns into being.

Of course, this does not mean that science should leave behind its inherent method of analysis or that we can undermine the importance of the role the brain plays. ${ }^{21}$

\footnotetext{
${ }^{20}$ As regards Dennett's criticism of the Chinese brain experiment see Dennett, 1980.

${ }^{21}$ Here I must note that Merleau-Ponty's caveat that scientific investigation inevitably bears some distortive potential (1967, p. 216) does not imply ignorance of scientific results. Rather, it calls attention to the difference between scientific and phenomenological approaches. The former aims at solving puzzles with regard to a welldefined phenomenon (e.g., an aspect of perception), while phenomenology attempts to reveal our primordial relation to our environment. Keeping in mind this distinction, we should be careful when drawing inferences.
} 
The mind, including conscious experience, arises from a web of relations that involve the brain, but the brain remains the hub in this web, in the sense that neural events contribute substantially to mental events and the brain has a role in orchestrating the relational web as its organizing center. (Adolphs, 2007, p. 875)

This orchestrating brain is slightly different as compared with RECS, as it ascribes the organizing potential rather to interactions between the organism and its environment. Regarding the brain as having this orchestrating function may conceal the importance of interactions with the external. Neuroscientists and neuropsychologists describe the brain as a predictive and learning ensemble of neural networks which learns in accordance with interactions with its environment. But despite this active intercourse with the environment, consciousness is thought to be related exclusively to the brain. Nonetheless, we can ask precisely what "would be internalized" if interactions in time, the "historical relations between the brain and environment" are ignored. (p. 874)

\subsection{Qualia in the brain?}

Similarly, the phenomenal character of a conscious experience is hardly explicable either in terms of neural patterns or restricted to internal processes. Graziano suggested that " $[\mathrm{m}] \mathrm{y}$ awareness feels like it is located inside me. In a sense it is me." If we consider awareness as the "perceptual model of attention", we can imagine that the monitoring of our own attention happens inside ourselves. Similarly, when Humphrey takes into account the mechanisms of sensation, we can easily relate it to a self in the present moment. Both cases remind us what consciousness meant at the turn of 17th and 18th centuries: consciousness is a self-reflective, self-referential knowledge/capability. But both models of consciousness are incapable of anchoring consciousness in the physical, or relate it to the external world.

As an alternative, sensorimotor approaches like RECS and phenomenology propose that consciousness is relational. In a nutshell ${ }^{22}$, O'Regan suggests a somewhat different terminology than Ned Block. He differentiates cognitive and conscious access. Having conscious access to something provides a wider context than cognitive access, because with conscious access it is also cognitively accessible that we have cognitive access to this something. That is, conscious access sets up a wider horizon regarding what to do next and enables a socially defined self. (At this point we can see an important difference as regards Cudworth's or Locke's comprehension.) O'Regan introduces raw feel (which is framed as something akin to qualia). Raw feel emerges out of active engagement with something in the world. "[F]eels should be conceived of as qualities of our mode of interaction with the world" (O'Regan, 2011, p. 110). Raw feel, unlike memories or imaginings, feels like something; it bears phenomenal character because being engaged with something in our environment provides us richness, bodiliness, partial insubordinateness, and grabbiness. This means we have a huge amount of accessible information (more than the engagement strictly needs), bodily movements entail perceptual changes, changes may occur independently from our movements, and salient changes grab our

\footnotetext{
${ }^{22}$ For a short outline of O'Regan's conception of conscious access and phenomenal consciousness, see Kondor (2015, pp. 153f.). For further details, see O’Regan (2011).
} 
attention. Raw feel has different qualities because it is constituted "by different modes of interaction" (p. 113). There is structure in the difference because raw feels are rooted in different activities; accordingly, there are differences between the laws that applied. Finally, raw feel is ineffable because we do not have cognitive access to all our movements.

Even researchers engaged in neuroscience and biology, such as Ralph Adolphs, accept Noë's and O'Regan's suggestion that our brain would be overloaded if we could not recline upon our environment, and suggest considering the body as emulator when we need to model other people's behavior. It is not only economical, but "[t]he body might be thought of as a 'somatic scratchpad' that we can probe with efferent signals in order to reconstruct knowledge about the details of an emotional state" (Adolphs, 2007, p. 875). The idea of the body as a somatic scratchpad proposes incorporating the body when cognitive processes are described. But Adolphs emphasizes the importance of the "interaction with the world", since otherwise we would not have change in conscious experience (p. 868). As with Nardi's action theory and RECS, Adolphs also considers "historical relations" as necessary.

\subsection{Evolution in the skull?}

Taking into account Dunbar's social brain hypothesis, considering the possibility of a hardwired capacity for social perception as Graziano understood it, and Humphrey's attempt at reconciling science and phenomenality from a functionalist perspective, we can ask how these capabilities could have evolved exclusively on an intra-cranial basis. Even the idea of evolution presupposes interaction, a symbiotic interplay between the organism and its environment.

As we can see, Donald considered cognitive evolution as being an encapsulating development of representational skills, with pictorial images paving the way for the creation of an external and lasting form of representation. ${ }^{23} \mathrm{He}$ proposes that "we are permanently wedded to our great invention, in a cognitive symbiosis unique in nature". (1993, p. 356) Thus, human minds can be considered as being hybridizations.

However, Donald's reconstruction of cognitive evolution is very close to the extended mind hypothesis ${ }^{24}$ as it suggests human cognition is externally scaffolded, and it does not go as far as the enactive approach of neuroarchaeology does.

$[\mathrm{N}]$ euroarchaeology is strictly an interactionist approach, aiming primarily to understand the bidirectional links between brains, minds and culture. Brain activity is a crucial component of the human mind but so is also material culture. The human mind as an interactive, embodied, and distributed autonoetic system is neither restricted to nor can it be identified with the temporal and spatial boundaries of the brain within (Malafouris, 2010, p. 64).

\footnotetext{
${ }^{23}$ External symbolic storage, of course, is meant as being public representation, i.e., accessible to others, as with mimicry and gestures. But the terminology, the theoretical background (Dunbar's conception of computing brain) and the focus on cognition place his theory closer to traditional than to recent radical branches of cognitive science.

${ }^{24}$ For more details, see Kondor (2006 esp. pp. 86-88).
} 
It highlights the importance of the interaction between the cognizing agent and its environment, or as Renfrew formulated: "[t]he mind works through the body." Accordingly, "[w]eight has first to be perceived as a physical reality —in hands and arms, not just in the brain within the skull—before it can be conceptualized and measured." (Malafouris, 2013, p. 33)

Similarly, via pictorial representations we gained "a new mode of epistemic access to the world of visual experience." Images as results of material engagements made the world accessible in a new way. "Through the process of 'imaging' the underlying mechanisms of human perception are being transformed to an object for perception and contemplation" (Malafouris, 2007, p. 299).

In this sense, neuroarcheology underscores the reciprocal relation between the artefact, its creators, and their mates; and emphasizes the importance of the physical body as the necessary starting point for any experience to feed cognition. Bodily skills and material engagements are in the focus of this approach, which does not consider pictorial representations as a result of "the existence of consciously manipulable content-bearing tokens, though it certainly sets up ... a visually stimulating channel of influence for their creation" (Malafouris, 2007, p. 293). Unlike Malafouris, Donald seems to believe that there is an underlying "linguistic conceptual skill” (Donald, 1993, p. 283).

In conclusion, I recommend extending the scope of investigation when cognition and consciousness are in question, because on the basis of phenomenological tradition, RECS' account of and sensorimotor's approach to consciousness provide a framework within which cognition and consciousness is tractable in a coherent framework, qualia is anchored both in the physical and subjective, and both cognition and consciousness can be considered in a timely expanded manner. Historically, the very idea of the incarnated/embodied mind as Merleau-Ponty explicated initiates an approach which embraces activity theory, RECS, and neuroarcheology (as Renfrew and Malafouris construed the term). It is in line with efforts eschewing representationalism and emphasizing cultural embeddedness, and calls attention to the distortive potential of scientific inquiry.

\section{Funding}

In the preparation of the present paper, I enjoyed the support of the Hungarian Scientific Research Fund K-112542.

\section{References}

Adolphs, R. (2007). Consciousness: Situated and Social. in Zelazo, P. D., Moscovitch, M. and Thompson, E. (eds.) The Cambridge Handbook of Consciousness, (pp. 863-878). Cambridge: Cambridge University Press.

Aizawa, K. (2015). What is this cognition that is supposed to be embodied? Philosophical Psychology 28 (6), 755-775, online available at: http://cepa.info/3949.

Chemero, A. (2009). Radical embodied cognitive science. Cambridge, MA: The MIT Press. 
De Jaeger, H., Di Paolo, E. and Gallagher, S. (2010). Can social interaction constitute social cognition? Trends in Cognitive Sciences, 14(10), 441-447.

Dehaene, S. (2014). Consciousness and the brain. Deciphering how the brain codes our thoughts. New York: Viking/Penguin Group.

Dennett, D. (1980). The Milk of Human Intentionality. Behavioral and Brain Sciences 3(3), 428430.

Dennett, D. (1995). The Unimagined Preposterousness of Zombies. Journal of Consciousness Studies, 2(4), 322-326.

Descartes, R. ([1644] 1985). The World or Treatise on Light. in The Philosophical Writings of Descartes. Vol. I. Cambridge: Cambridge Univ. Press.

Descartes ([1661] 1995). Mediattions on First Philosophy. in The Philosophical Writings of Descartes, [1-62]. Vol.II. Cambridge: Cambridge Univ. Press.

Donald, M. ([1991] 1993). Origins of the Modern Mind. Three Stages in the Evolution of Culture and Cognition, Cambridge, MA: Harvard Univ. Press.

Dunbar, R. I. M. (1998). The Social Brain Hypothesis. Evolutionary Anthropology. 6 (5), 178-190.

Fiebich, A., Gallagher, S and Hutto, D. D. (2016). Pluralism, Interaction, and the Ontogeny of Social Cognition. in Kiverstein, J. (Ed.) The Routledge Handbook of Philosophy of the Social Mind. London: Taylor \& Francis Ltd.

Graziano, M.S.A. \& Kastner, S. (2011). Human consciousness and its relationship to social neuroscience: A novel hypothesis. Cognitive Neuroscience, 2(2), 98-133.

Graziano, M.S.A. (2014). Speculations on the Evolution of Awareness. Journal of Cognitive Neuroscience, 26(6), 1300-1304.

Heidegger, M. ([1927]1962). Being and Time. Oxford: Basil Blackwell.

Humphrey, N. (2000). How to Solve the Mind-Body Problem. Journal of Consciousness Studies, 7 (4), $5-20$

Humphrey, N. (2008). Seeing Red. Cambridge, MA: Harvard University Press.

James, W. (1884). What is Emotion? Mind, 9, (34), 188-205.

James, W. ([1904] 1987). Does 'consciousness' exist? In James, William James: Writings 19021910 (pp. 1141-1158). New York: Library Classics of the United States.

James, W. ([1890] 1932). The principles of psychology: Vol. I. New York: Henry Holt and Company.

Kiverstein, J. (2016). The Interdependence of Embodied Cognition and Consciousness. Journal of Consciousness, 23(5-6), 105-137.

Kondor, Z. (2003). Changing Media: Perennial Challenge for Philosophy. in Nyíri, K. (Ed.), Mobile Learning. Essays on Philosophy, Psychology and Education, (pp. 69-79) Vienna: Passagen Verlag.

Kondor, Z. (2006). Being Mobile: Cognitive Multiplicity. in Nyíri, K. (Ed.), Mobile Understanding. The Epistemology of Ubiquitous Communication, (pp. 79-90), Vienna: Passagen Verlag. 
Kondor, Z. (2015). Theoretical Controversies - Terminological Biases: Consciousness Revisited. Studies in Logic, Grammar and Rhetoric, 41 (54), 143-160.

Locke, J. ([1690] 1999). An Essay Concerning Human Understanding. Pennsylvania State University, Electronic Classics Series.

Malfouris, L. (2007). Before and Beyond Representation: Towards an Enactive Conception of the Palaeolithic Image. in Renfrew, C. and Morley, I. (Eds.) Image and imagination: A Global Prehistory of Figurative Representation (pp. 289-302). Cambridge: McDonald Institute of Archaeological Research,.

Malafouris, L. (2010). Metaplasticity and the human becoming: principles of neuroarchaeology. Journal of Anthropological Sciences, 88 (4), 49-72.

Malafouris, L. (2013). How Things Shape the Mind. A Theory of Material Engagement. London: The MIT Press.

Matlin, M. W. (2013). Cognition. Danvers, MA: John Wiley \& Sons, Inc.

Merleau-Ponty, M. ([1942] 1967). The structure of behavior. Boston: Beacon Press

Merleau-Ponty, M. ([1945] 2005). Phenomenology of perception. London, UK: Routledge.

Merleau-Ponty, M. (1964). The primacy of perception. Evanston: Northwestern Univ. Press.

Nardi, B. A. (1996). Concepts of Cognition and Consciousness: Four Voices. in B. A. Nardi (Ed.) Context and Consciousness: Activity Theory and Human-Computer Interaction (pp. 64-79). Cambridge: MIT Press.

O'Regan, K. (2011).Why red doesn't sound like a bell? Oxford, UK: Oxford Univ. Press.

Schmal, D., (2012) A kezdet nélküli kezdet. Descartes és a kartezianizmus problémája. Budapest: Gondolat.

Silberstein, M. \& Chemero, A. (2012). Complexity and extended phenomenological-cognitive systems. Topics in Cognitive Science, 4(1), 35-50.

Sperry, R. W. (1969). A Modified Concept of Consiousness. Psychological Review, 76 (6), $532-$ 536

Thiel, U. (1991). Cudworth and Seventeenth-Century Theories of Consciousness. In Gaukroger, S. (Ed.), The Uses of Antiquity. The Scientific Revolution and the Classical Tradition, (pp. 79-99). Dordrecht: Springer

Van Gelder, T. (1999). Wooden Iron? Husserlian Phenomenology Meets Cognitive Science, in J. Petotot, F.J. Varela, B. Pachoud (eds), Naturalizing Phenomenology. Issues in Contemporary Phenomenology and Cognitive Science. (pp. 245 - 265). Stanford: Stanford Univ. Press.

Wittgenstein, L. (1958). The Blue and Brown Books. Oxford: Basil Blackwell

The editorial and publishing process of this publication has been financed by the Ministry of Science and Higher Education from the funds for the dissemination of research (DUN) within the framework of publishing activity, contract no. 711/P-DUN/2019, period of implementation: the years 2019-2020. 